\title{
Fusion of human umbilical cord mesenchymal stem cells with esophageal carcinoma cells inhibits the tumorigenicity of esophageal carcinoma cells
}

\author{
YONG WANG $^{1 *}$, HONGBIN FAN $^{1 *}$, BING ZHOU $^{2}$, ZHENYU JU ${ }^{1}$, LONG YU $^{1}$, \\ LIPING GUO $^{1}$, JINGDONG HAN ${ }^{2}$ and SHIXIN LU ${ }^{1}$ \\ ${ }^{1}$ Department of Etiology and Carcinogenesis, Chinese Academy of Medical Sciences and Peking \\ Union Medical College; ${ }^{2}$ Chinese Academy of Sciences, Beijing, P.R. China
}

Received July 3, 2011; Accepted August 16, 2011

DOI: 10.3892/ijo.2011.1232

\begin{abstract}
Prior studies on the biology and therapeutic application of human stem cells in human malignancies have reported mixed results. Some evidence shows the use of stem cell transplantation is an important tool in the treatment of several hematologic and non-hematologic malignancies while some others suggest both human stem cells and mature stromal cells can contribute to the development and growth of human malignancies. Aiming to provide more evidence on this controversial issue, we investigated the effect of cell fusion of mesenchymal stem cells with esophageal carcinoma cells on tumorigenesis. Results suggest that artificial fusion of human umbilical cord mesenchymal stem cells with esophageal carcinoma cells resulted in hybrids with declined cell growth, increased apoptosis and suppressed tumorigenicity. The comparison of gene expression profiles of human mesenchymal stem cells, esophageal carcinoma cells and hybrids indicated that fusion induced activation of apoptosis. Furthermore, the expression of DUSP6/MKP3 in MAPK pathway increased strikingly and the exogenous overexpression confirmed the growth suppression. Our results demonstrate fusion of human mesenchymal stem cells with esophageal carcinoma cells induced apoptosis and benign transdifferentiation rather than reprogramming to cancer stem cells.
\end{abstract}

\section{Introduction}

Work over the past few decades has greatly improved our understanding of the biology and therapeutic application of

Correspondence to: Dr Shixin Lu, Department of Etiology and Carcinogenesis, Chinese Academy of Medical Sciences and Peking Union Medical College, Beijing, P.R. China

E-mail: shlu@pubem.cicams.ac.cn

*Contributed equally

Key words: cell fusion, mesenchymal stem cells, esophageal carcinoma cells, apoptosis, DUSP6/MKP3, reprogramming human stem cells in human malignancies. The use of stem cell transplantation is shown to be an important tool in the treatment of several hematologic and non-hematologic malignancies (1-3). Systemic administration of untreated or genetically modified mesenchymal stem cells (MSCs) could target multiple tumor sites and exert potent antitumorigenic effects in several tumor models with no evidence of metastasis or recurrence (4-7). However, several studies have altered the perception of the stromal cells that surround epithelial tumors from being innocent bystanders in the neoplastic process to being a cell type that actively promotes the growth and invasion and metastasis of the adjacent transformed cells (8-10). We aim to provide more evidence on this controversial issue.

MSCs were identified based on their ability to differentiate into mesenchymal lineage cells and to express specific markers (11). In the 1970's, a serial systematic experiments proposed that fusion between cancer cells and normal cells tended to abrogate the malignant phenotype (12-14). Recent reports indicated that fusion could be a novel mechanism for cell repair and stem cell differentiation (15-17). However, the prior work showed that effect of cell fusion of MSCs with tumor cells on tumorigenesis is still slightly ambiguous.

To explore the role of cell fusion of MSCs with tumor cells on tumorigenesis, we studied the effects of human MSCs (hMSCs) on the esophageal carcinoma (EC) because it occurs with high prevalence in many areas of the world especially in China $(18,19)$.

Our results revealed that cell fusion significantly suppressed tumorigenicity in vitro and in vivo. Further investigation suggested that fusion-induced apoptosis, overexpression of DUSP6/ MKP-3 and transdifferentiation could be the mechanism of the inhibition.

\section{Materials and methods}

Cell cultures. Human esophageal cancer cell line EC9706 was established by the Cancer Institute, Chinese Academy of Medical Science, KYSE150 was kindly provided by Dr Shimada. Both cell lines were cultured according with their original methods. HMSCs and commercial media MSCM were purchased from AmCellGene Co. Ltd. (Tianjin, China). Both stem and fusion 
cells were cultured in MSCM and changed every 3 days; cells were trypsinized with TrpLE (Gibco, Carlsbad, CA, USA) and split when $80 \%$ confluence was achieved. All hMSCs were used at passage 3-12 for all experiments.

Cell fusion, sorting and identification. Heterokaryons were generated by fusing DiD-labeled hMSCs and DiO-labeled ECs with PEG1500 (Roche, Indianapolis, IN, USA) according to the method previously described (20). After 3 passages, cell immunophenotyping was performed as described with antibodies: CD90-FITC, CD105-PE, isotype control mouse IgG1-FITC and IgG1-PE (BioLegend, San Diego, CA, USA) (21). CK18 expression was investigated as described (22). The dilution ratio of antibody CK18 was 1:200; TRITC-conjugated IgG was 1:1000 (Santa Cruz, Santa Cruz, CA, USA). Cell nuclear was counterstained with $2 \mu \mathrm{g} / \mathrm{ml}$ DAPI (Sigma, St. Louis, MO, USA). Images were acquired by BX51epi-fluorescent microscope (Olympus, Tokyo, Japan) and processed with Photoshop CS 5 (Adobe, San Jose, CA, USA).

Soft agar colony formation assay. Aliquots of logarithmically growing cells (1000 cells) in single cell suspensions were seeded in 6-well plates with complete medium for soft agar colony formation, 2000 cells were suspended as single cells in soft agar (Sigma) and the numbers of colonies were quantified as described (22).

Xenograft assays in immunodeficient mice. SCID mice were purchased from Vital-river Co. (Beijing, China). Female athymic nude mice (Balb/c nu/nu) were obtained from the Institute of Laboratory Animal Science (Beijing, China). All experiments were approved by the animal care committee of Chinese Academy of Medical Sciences \& Peking Union Medical College and performed as previously described (22).

Oligonucleotide microarray analysis. Total RNA was extracted from fusion cells, MSCs, and EC9706 using TRIzol reagent (Invitrogen) according to the manufacturer's instructions. Microarray analysis was performed following a standard protocol (CapitalBio Corp., China). Fluorescently labeled (Cy5 and Cy3-dCTP) cDNA was produced using Eberwien's linear RNA amplification method and subsequent enzymatic reaction. To measure technical replication, a one swap-dye experiment was performed on each of the biological samples. Arrays were scanned with a confocal LuxScan ${ }^{\mathrm{TM}}$ scanner and the images obtained were analyzed using LuxScan ${ }^{\mathrm{TM}} 3.0$ software (both from CapitalBio). A space- and intensity-dependent normalization based on the LOWESS program was performed.

Apoptosis assays. Cell viability was determined by MTT (Sigma), cell apoptosis were detected using Annexin-V and PI (BD, San Jose, CA, USA), followed by flow cytometric analysis. Subcellular apoptosis structure was identified with Philips EM208s transmission electron microscope (Eindhoven, The Netherlands) by experts at the Electron Microscopy Core Facility (CAMS-PUMC, Beijing, China).

Transfection experiments. The DUSP6 plasmids (pCMVDUSP6) and empty vector (pCMV-AC) were purchased from OriGene (Rockville, MD, USA). Cells were transfected using
Lipofectamine (Invitrogen, Carlsbad, CA, USA) and the stable clone was screened with G418. The transient or stable transfected cells were harvested at a proper time for survival, apoptosis and tumorigenesis assays.

Quantitative real-time PCR. Total RNA extraction, treatment, cDNA synthesize and real-time PCR were performed as described (24). Primers were designed and validated by Primer-Blast to guarantee the pairs were separated by at least one intron on the corresponding genomic DNA. Fold changes were calculated using 2- $\Delta \Delta \mathrm{CT}$ method (25). GAPDH was used as internal control. The DUSP6 cDNA primers used for PCR were 5'-GCAATACTTTGGGTTGGTTTCT-3' (forward) and 5'-ACACCACGAACATAATGGAGAA-3' (reverse). The final product length was $147 \mathrm{bp}$. The GAPDH cDNA primers used for PCR were 5'-TGTTGCCATCAATGACCCCTT-3' (forward) and 5'-CTCCACGACGTACTCAGCG-3' (reverse). The final product length produced using these primers was $202 \mathrm{bp}$.

Western blot analysis. Western blotting was performed as described (22). The dilution ratio of antibodies was 1:1000 for anti-DUSP6 (OriGene), 1:5000 for anti- $\beta$-actin, 1:5000 for HRP-conjugated IgG (Santa Cruz). The protein bands were visualized by ECL (Pierce, Rockford, IL, USA) and recorded on X-film (Kodak, Rochester, NY, USA) or photographed with ImageQuant LAS 4000 (GE Healthcare, Piscataway NJ, USA). $\beta$-actin was used as loading control.

Osteogenic and adipogenic differentiation. Fusion cells and hMSCs were plated in 6-well plates at a density of $2 \times 10^{4}$ cells per well. Specific induction medium (Invitrogen) was added $24 \mathrm{~h}$ later. After 3 weeks of induction, cells were stained using the von Kossa procedure or oil red solution to detect the presence of calcium deposition in osteocytes or neutral lipid vacuoles in adipocytes (21).

Statistical analysis. The quantitative results were represented as the means \pm SD unless otherwise stated. For comparison, first the normal distribution was tested then proper 2-tailed method was chosen accord with the experimental design. A 95\% ( $<<0.05)$ confidence interval was applied for statistical significance. Data were analyzed with SPSS18.0 (SPSS Inc., Chicago, IL, USA) and plotted with Prism 5 (GraphPad Inc., San Diego, CA, USA).

\section{Results}

Cell fusion and isolation of hybrids. According to the experimental strategy (Fig. 1A), fusion partners were stained with the lipophilic fluorescent dyes $\mathrm{DiO}$ or $\mathrm{DiD}$ and maximum double stained hybrids were found at $24 \mathrm{~h}$ post-fusion (Fig. 1B). In order to identify the hybrids we observed double stained binucleated or multinucleated hybrids with confocal one day post-fusion. The results in Fig. 1D indicated the successful fusion and it was re-affirmed by the TEM (Fig. 2De and f). After fluorescence activated cell sorting (FACS, Fig. 1B), the enriched heterokaryons were cultured $\sim 15-30$ days for colonies formation. Most hybrids died in one week and each survival colony was picked up for further screening. Stem cell markers CD90, CD105 and epithelia marker CK18 were chosen to identify the cell origin (22). Finally, three $\mathrm{CD}^{+}{ }^{+}, \mathrm{CD}_{105}{ }^{+}$and $\mathrm{CK} 18^{+}$clones from EC9706-hMSCs 


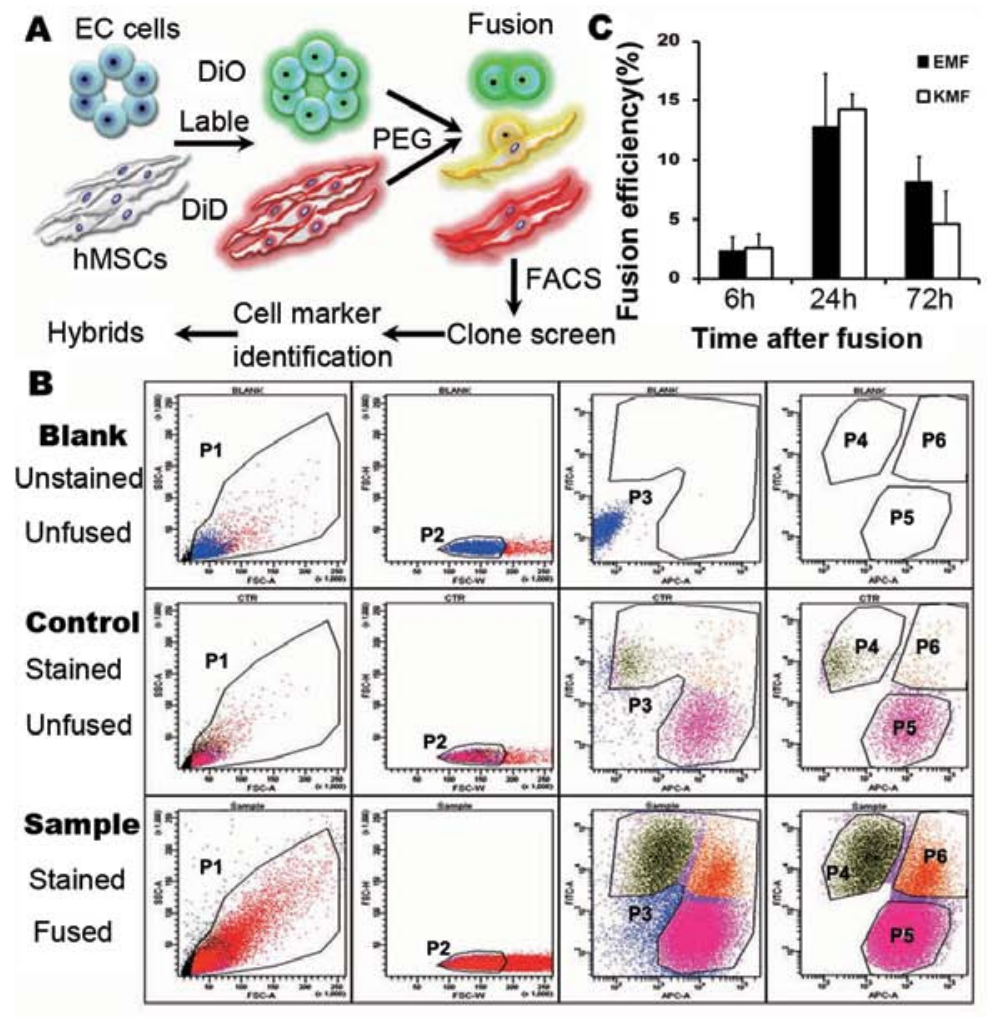

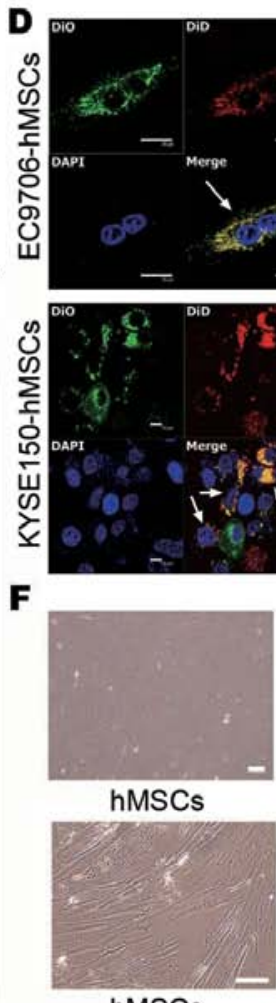

hMSCs
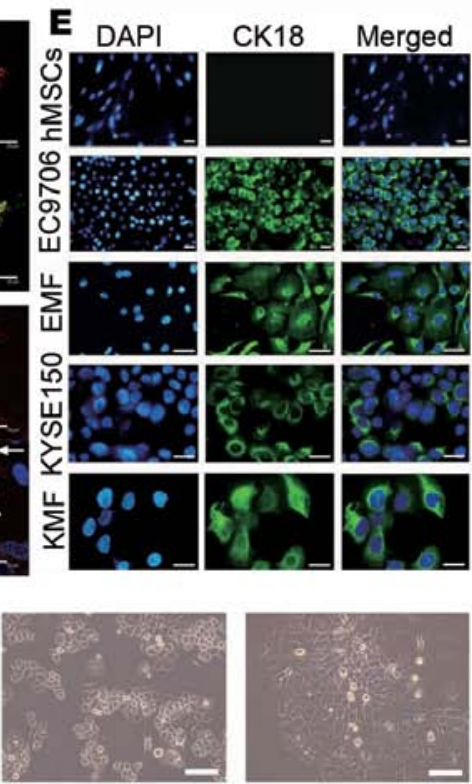

EC9706

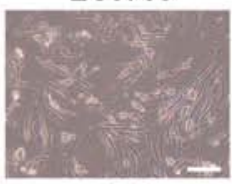

EMF

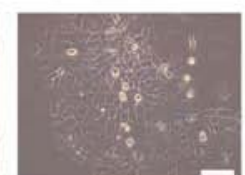

KYSE150

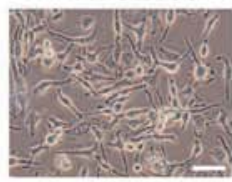

KMF

Figure 1. Establishment and identification of fusion cells. (A) Experimental strategy used to generate heterokaryons. (B) Flow cytometry analysis and sorting of hybrids. The blank group was a mixture of unstained fusion partners and the mock fused stained cells were the control group. Double positive hybrids of different samples were sorted in gate P6 and single positive self-fusion cells were collected in gate P4 or P5. (C) Fusion efficiency assay. The percentage of double positive cells against the total number of fusion partners was analyzed at 6,24 and $72 \mathrm{~h}$ post-fusion $(\mathrm{n}=3)$. The maximum efficiency was at $24 \mathrm{~h}$ and set for the efficient sorting time. The number of false-positive cells was noted and subtracted in all calculations. (D) Representative confocal image of hybrids. Upper box, EC9706 cell fused with hMSCs. Scale bar, $20 \mu \mathrm{m}$. Magnification, x600. Lower box, KYSE150 cell fused with hMSCs. Scale bar, $10 \mu \mathrm{m}$. Magnification, x400. Cell membrane structures were stained with $\mathrm{DiD}$ (red) or $\mathrm{DiO}$ (green); cell nuclei were stained with Hoechst 33342 (blue); hybrids are yellow and multinuclear (arrow). (E) The expression of epithelial cells marker CK18. Monolayer cell cultures were stained by a CK18 antibody with the secondary florescent antibody (green). Cell nuclei were stained with DAPI (blue). CK18 was negative in hMSCs, but positive in ECs and hybrids. Bars, $20 \mu \mathrm{m}$. Magnification, hMSCs x200, EC9706 x200, EMF x400, KYSE150 x400 and KMF x400. (F) Morphological changes after fusion. Micrographs show the hMSCs with fibroblast-like growth; ECs show epithelial growth; hybrids grew with the characteristics of both parents. Bars, $100 \mu \mathrm{m}$. Magnification, hMSCs x200, others x400.

were isolated and nominated EMF1-3; three $\mathrm{CD}_{105^{+}}$and $\mathrm{CK} 18^{+}$ clones from KYSE150-hMSCs were named KMF1-3 (Table I). The hybrids expressed both parental markers (Fig. 1E) and acquired fibroblast-like morphology (Fig. 1F), implying the transdifferentiation might be induced by fusion.

Cell fusion inhibits tumorigenicity. In order to evaluate the tumorigenic potential of the hybrids, we compared their ability of anchorage-independent growth in vitro at first. The hMSCs could not form colonies in soft agar and colonies of hybrids remarkably decreased comparing with ECs (Fig. 2A and $\mathrm{B})$. Then we examined the tumorigenesis in vivo with SCID mice engraftments. All mice formed tumors in EC9706 group. Although 2/6 of EMF1, 4/6 of EMF2 and 6/6 mice formed tumors in EMF3, the volume and weight of tumors in fusion groups were significantly smaller than those of EC9706 (Table II). Pathology results confirmed that all the tumors were esophageal squamous carcinoma. To prove these results, fusion of hMSCs-ECs were repeated and self-fusion cells were collected as controls (same cell culture conditions). Both ECs and their self-fusion groups developed tumors. No tumor formed in hMSCs or their self-fusion group within a prolonged waiting time. Although 72\% (13/18) mice of EMFs and 61\% (11/18) of KMFs developed tumors, the tumor latency time increased and weight and volume decreased, compared to that of ECs or their self-fusion groups. Furthermore, spontaneous apoptosis of hybrids was analyzed. Results showed that apoptosis indexes of hybrids were about twice that of their parents (Fig. 2C). Meanwhile, the morphologic characteristics of apoptosis, such as cytoplasm clouding, cell shrinkage, nuclear condensation and fragmentation, were confirmed by TEM (Fig. 2D).

Gene expression analysis by microarray. To further understand the gene expression differences before and after cell fusion, and the mechanism of tumor suppression, we compared transcriptional profiles of EC9706, hMSCs and EMFs. A total of 4548 significantly $(\mathrm{p}<0.05)$ differentially expressed genes (DEGs) among the three cell lines were found by LIMMA. Functional annotation and pathway enrichment predicted that DNA damage repair, cell cycle arrest and apoptosis pathways, protein synthesis or transportation and cell proliferation pathways were significantly changed in EMFs than hMSCs and/ or ECs. Furthermore, total DEGs were mapped to the canonic pathways with KEGGanim. Fig. 3 depicts the core genes of 

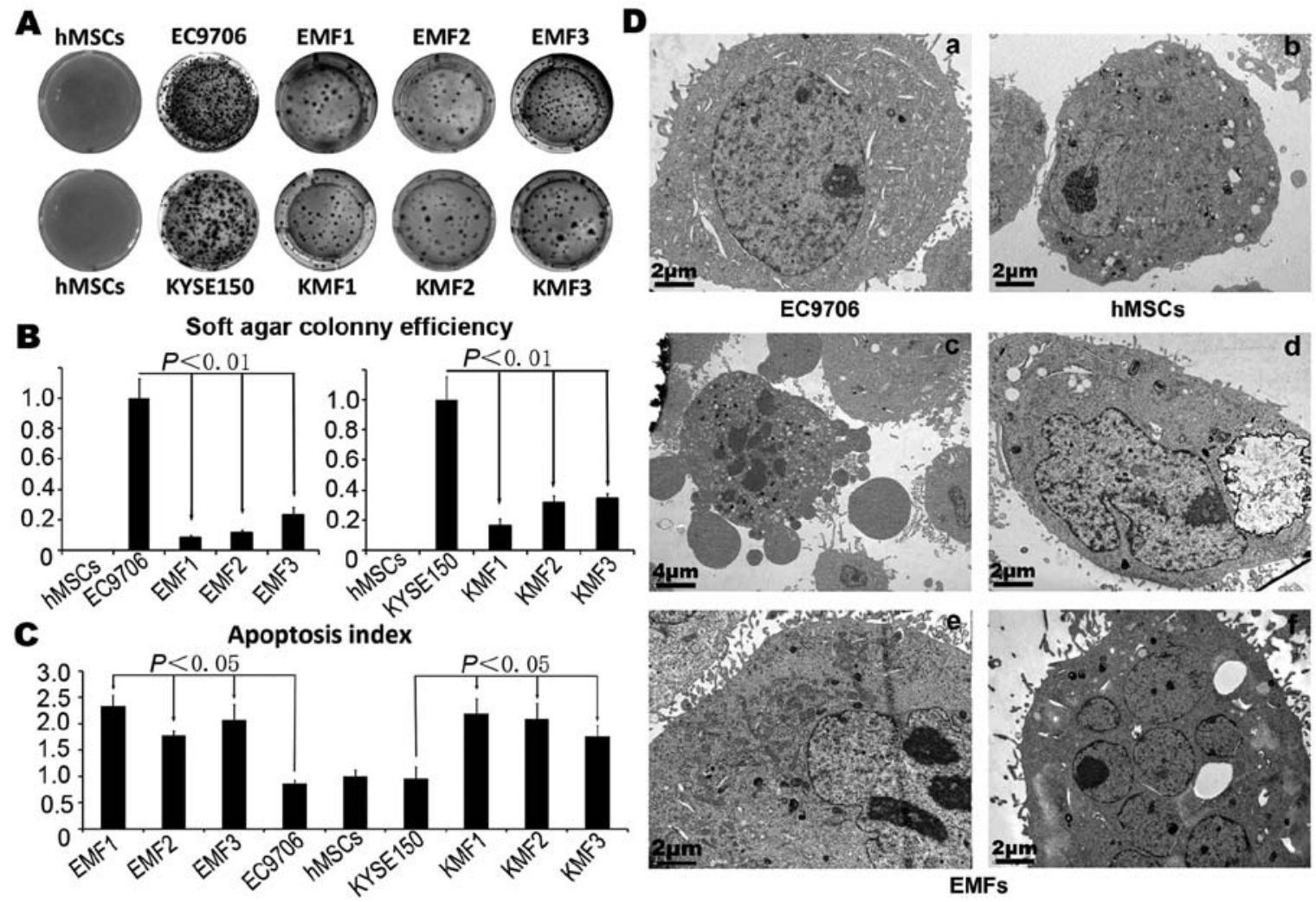

Figure 2. Colony formation and apoptosis assay. (A) Representative soft agar colony images of hybrids. The passage of hMSCs was at 6, EMFs was at 5, and KMFs were at passage 8. (B) Colony formation efficiency. There was no colony in the hMSC group and the colony number of ECs was arbitrarily set at 1 (n=3). (C) Quantification of apoptosis. The annexin V positive stains represent the early and late stage of apoptotic cells and the index of hMSCs was arbitrarily set at 1 (n=3). (D) Typical transmission electron micrographics. (a) EC9706 has a big rounded nuclear. (b) hMSCs have the normal N/C ratio and ameboid nuclear morphology. (c-f) EMFs. Pictures show typical apoptosis bodies and mitochondrial swelling (c), cytoplasm clouding and vacuolated (d), cell shrinkage and nuclear condensation changes of spontaneous apoptosis (e and f). It also displayed multinuclear or multinucleolar hybrids, the N/C ratio was increased and nuclei were ameboid folded like hMSCs, which confirmed the cell fusion (e and f). Scale bars are labeled, Magnification, c x8000, others x15000.

Table I. Characterization of hMSC surface markers.

\begin{tabular}{lcc}
\hline Strain & CD90 & CD105 \\
\hline hMSCs & $99.9 \pm 0.01 \%$ & $99.8 \pm 0.01 \%$ \\
EC9706 & $0.6 \pm 0.02 \%$ & $99.8 \pm 0.01 \%$ \\
EMF1 & $40.1 \pm 5.32 \%$ & $93.5 \pm 0.02 \%$ \\
EMF2 & $69.9 \pm 8.26 \%$ & $96.8 \pm 0.27 \%$ \\
EMF3 & $46.7 \pm 10.56 \%$ & $95.0 \pm 2.37 \%$ \\
KYSE150 & $0.8 \pm 0.02 \%$ & $3.2 \pm 0.01 \%$ \\
KMF1 & $0.3 \pm 0.01 \%$ & $59.2 \pm 7.58 \%$ \\
KMF2 & $0.7 \pm 0.01 \%$ & $59.5 \pm 6.74 \%$ \\
KMF3 & $0.4 \pm 0.02 \%$ & $30.1 \pm 11.28 \%$
\end{tabular}

The quantitative results are presented as the mean $\pm \mathrm{SD}$ and the percentage was average of passages $2,5,10$ of each cell line.

DNA damage repair, cell cycle arrest and pro-apoptosis that were upregulated and might couple together to respond to fusion induced damage stress. The upregulation of negative factor DUSP6 of the MAPK pathway might inhibit the positive signals after fusion.
Tumor suppressor activity of DUSP6. Microarray analysis implied DUSP6 was the key regulator in MAPK pathway and their expression were confirmed by real-time PCR and Western blotting (Fig. 4A). Hence, we overexpressed full length DUSP6 to investigate the possible suppressive function in ECs. After transfection, DUSP6-overexpressed ECs (Fig. 4B) survival decreased (Fig. 4C) and apoptosis increased (Fig. 4D) markedly. All DUSP6-overexpressed KYSE150 died in 2 weeks in stable screening with $100 \mu \mathrm{g} / \mathrm{ml} \mathrm{G} 418$. In the case of transient transfection, all DUSP6 overexpressed ECs displayed a marked reduction of soft agar colony forming (Fig. 4E and F) and tumor formation ability (Table III). The stable clones of DUSP6-overexpressed groups had similar results in soft agar colony forming (Fig. 4A, E and F and Table III). In short, tumorigenesis was remarkable depressed through overexpression of DUSP6 in ECs.

Differentiated potential of hybrids. Reprogramming potential of MSCs has been confirmed by their phenotype adaptation after fusion with the recipient cells (24). Therefore, induced differentiation experiment was used to identify the differentiation potency, however, the results showed the hybrids did not inherit the osteogenic (Fig. 5A) or adipogenic potential from hMSCs (Fig. 5B). Hybrids did not acquire the multi-lineage differentiation potential of hMSCs. 
Table II. Tumor formation of hybrids in immunodeficient mice.

\begin{tabular}{|c|c|c|c|c|c|c|c|c|}
\hline Mouse & Groups & $\begin{array}{l}\text { Inoculate } \\
\text { cell no. }\end{array}$ & $\begin{array}{l}\text { Tumors/ } \\
\text { mice }\end{array}$ & Weight (mg) & Volume $\left(\mathrm{mm}^{3}\right)$ & $\begin{array}{c}\text { Tumor latency } \\
\text { (days) }\end{array}$ & $\begin{array}{l}\text { Sacrifice time } \\
\quad \text { (days) }\end{array}$ & P-value \\
\hline \multirow[t]{4}{*}{ SCID } & (1)EC9706 & $5 \times 10^{5}$ & $6 / 6$ & $1291.7 \pm 348.6$ & $601.4 \pm 104.3$ & $9.3 \pm 1.0$ & 35 & \\
\hline & (2)EMF1 & $5 \times 10^{5}$ & $2 / 6$ & $30.0 \pm 14.1$ & $7.8 \pm 1.3$ & 27 & 35 & a \\
\hline & (3)EMF2 & $5 \times 10^{5}$ & $4 / 6$ & $32.5 \pm 12.6$ & $17.7 \pm 7.8$ & 27 & 35 & a \\
\hline & (4)EMF3 & $5 \times 10^{5}$ & $6 / 6$ & $305.0 \pm 119.5$ & $118.2 \pm 67.8$ & $16.7 \pm 4.1$ & 35 & a \\
\hline
\end{tabular}

$\mathrm{Balb} / \mathrm{c} \mathrm{nu} / \mathrm{nu}$

\begin{tabular}{|c|c|c|c|c|c|c|c|}
\hline (5)hMSCs & $1 \times 10^{6}$ & $0 / 7$ & ND & ND & ND & 22 & \\
\hline (6)MMF & $1 \times 10^{6}$ & $0 / 5$ & ND & ND & ND & 51 & \\
\hline (7)KYSE150 & $1 \times 10^{6}$ & $6 / 6$ & $175.7 \pm 88.4$ & $329.5 \pm 160.0$ & $7.3 \pm 1.8$ & 22 & \\
\hline (8)KKF & $1 \times 10^{6}$ & $5 / 5$ & $286.7 \pm 121.6$ & $402.2 \pm 195.7$ & $10.6 \pm 1.6$ & 32 & \\
\hline (9)KMF1 & $1 \times 10^{6}$ & $2 / 6$ & $37.9 \pm 31.3$ & $58.5 \pm 43.5$ & $16.5 \pm 5.5$ & 22 & $\mathrm{~b}$ \\
\hline (10)KMF2 & $1 \times 10^{6}$ & $5 / 6$ & $21.4 \pm 4.4$ & $26.6 \pm 4.1$ & $19 \pm 1.0$ & 22 & b \\
\hline (11)KMF3 & $1 \times 10^{6}$ & $6 / 6$ & $58.4 \pm 32.2$ & $69.2 \pm 48.5$ & $18.7 \pm 0.9$ & 22 & $\mathrm{~b}$ \\
\hline (12)EC9706 & $1 \times 10^{6}$ & $6 / 6$ & $347 \pm 64.5$ & $448.2 \pm 148.3$ & $5.5 \pm 1.5$ & 22 & \\
\hline (13)EEF & $1 \times 106$ & $5 / 5$ & $412.6 \pm 89.2$ & $516.7 \pm 174.1$ & $8.3 \pm 3.5$ & 32 & \\
\hline (14)EMF1 & $1 \times 10^{6}$ & $2 / 6$ & $3.5 \pm 0.6$ & $3.1 \pm 0.1$ & 22 & 22 & c \\
\hline (15)EMF2 & $1 \times 10^{6}$ & $4 / 6$ & $8.8 \pm 2.4$ & $9.7 \pm 2.0$ & 22 & 22 & c \\
\hline (16)EMF3 & $1 \times 10^{6}$ & $5 / 6$ & $63.9 \pm 58.3$ & $95.0 \pm 76.0$ & $8.4 \pm 4.5$ & 22 & c \\
\hline
\end{tabular}

MMF, KKF and EEF means self-fusion of hMSCs, KYSE150 or EC9706 respectively. ND, not detected. Wilcoxon signed ranks test, significant difference of tumor weights, volumes and the latency times between groups. ${ }^{\mathrm{a}} \mathrm{p}<0.01$ (vs. group 1 ); ${ }^{\mathrm{b}} \mathrm{p}<0.01$ (vs. group 7 or 8 ); ${ }^{\mathrm{c}} \mathrm{p}<0.01$ (vs. group 12 or 13).

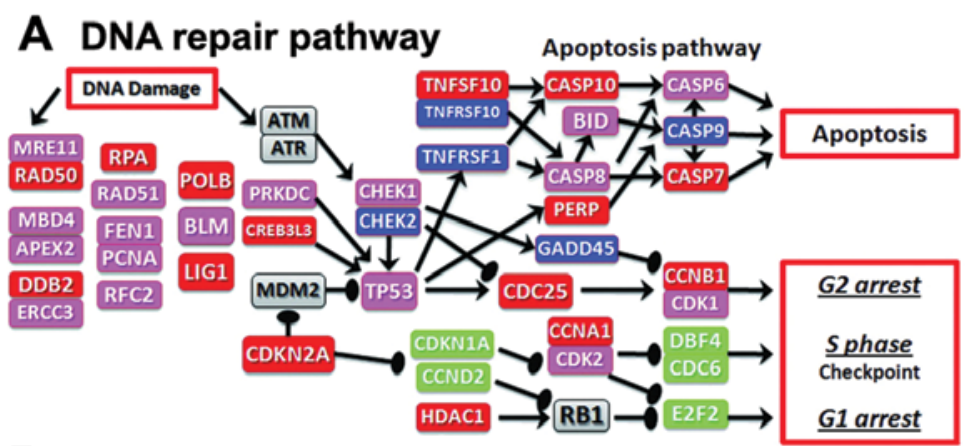

B

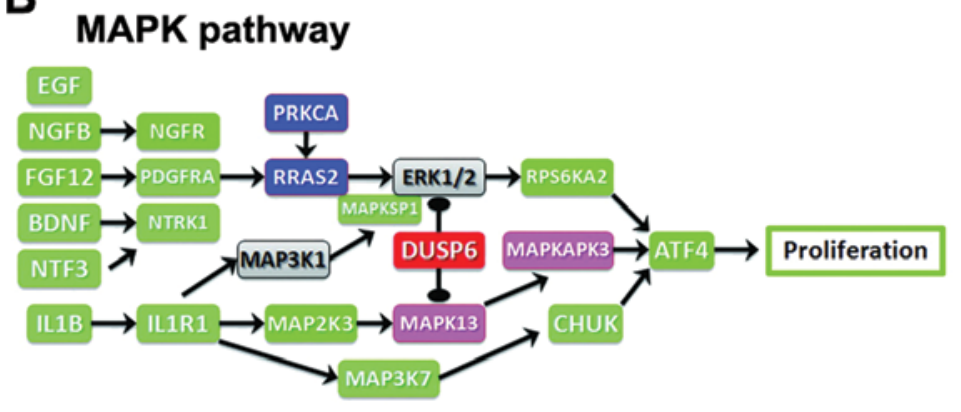

\begin{tabular}{|c|c|}
\hline GENE & $\begin{array}{l}\text { DEGs higher than } \\
\text { EC and hMSC }\end{array}$ \\
\hline GENE & $\begin{array}{l}\text { DEGs higher than } \\
\text { EC or hMSC }\end{array}$ \\
\hline GENE & $\begin{array}{l}\text { DEGs fall in between } \\
\text { EC and hMSC }\end{array}$ \\
\hline GENE & $\begin{array}{l}\text { DEGs lower than } \\
\text { EC or hMSC }\end{array}$ \\
\hline GENE & $\begin{array}{l}\text { DEGs higher than } \\
\text { EC and hMSC }\end{array}$ \\
\hline GENE & non DEGs \\
\hline$\rightarrow$ & activation \\
\hline$\rightarrow$ & inhibition \\
\hline & $\begin{array}{l}\text { increased bio-function } \\
\text { or process }\end{array}$ \\
\hline & intermediate state \\
\hline & $\begin{array}{l}\text { decreased bio-function } \\
\text { or process }\end{array}$ \\
\hline
\end{tabular}

Figure 3. Microarray analysis. Recapitulatory signaling pathway of DEGs. Log2 ratio of average DEG expression datasets was uploaded to the KEGGanim, a web-based tool for visualizing dynamic changes over cell fusion. The simplified hand-drawn model picture depicts the highlights of important sub-pathways and their potential biological functions or changed processes. (A) Core genes of DNA damage repair, cell cycle arrest, and p53 pathway were upregulated to induce apoptosis. (B) Negative feedback factor DUSP6 in MAPK pathway was upregulated to count back the positive signalling of cell proliferation. 
A

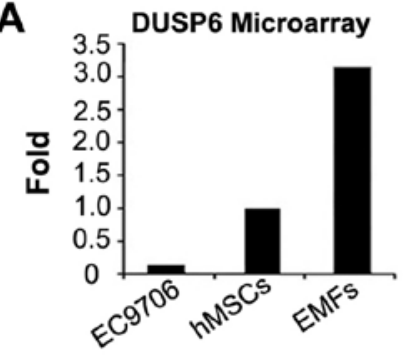

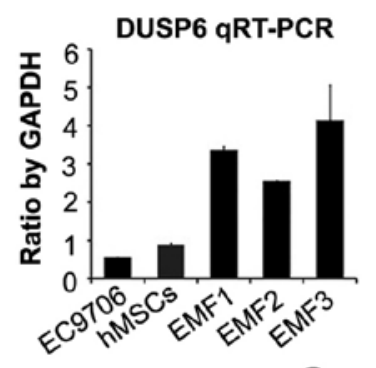

DUSP6 Western blot

B

Transient transfection

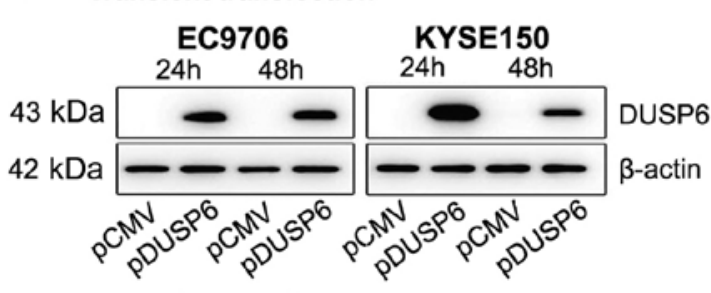

Stable transfection

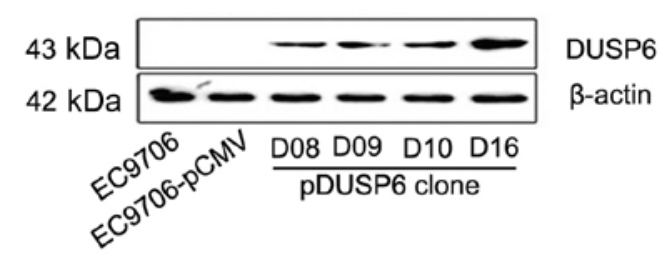

E

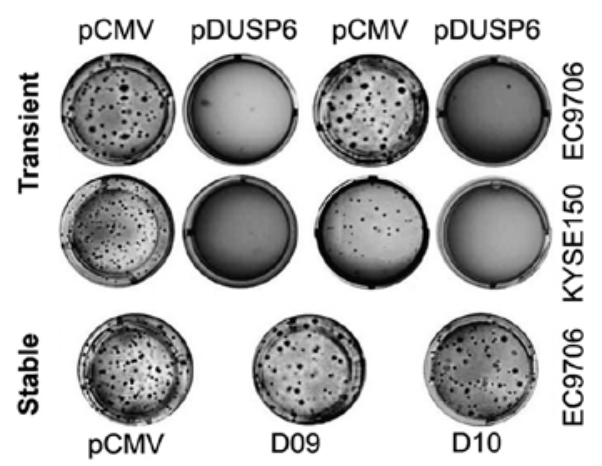

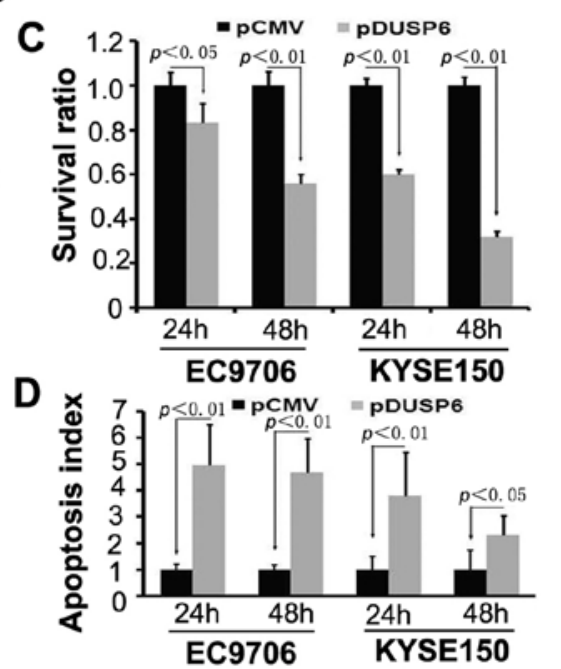

F

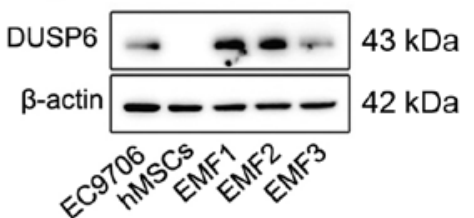

EC9706 KYSE150

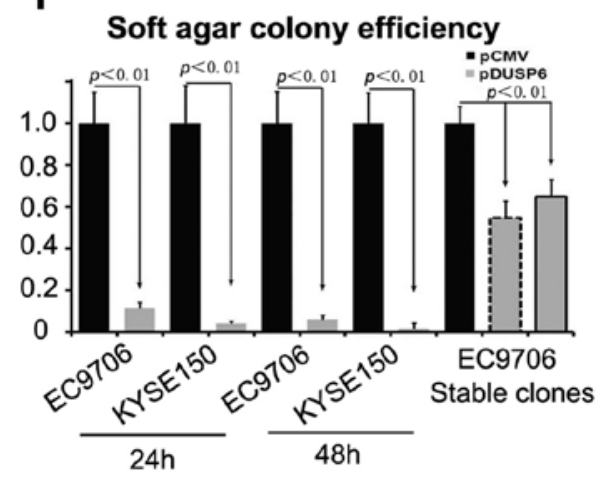

Figure 4. Exogenous overexpression of DUSP6 in EC cells. (A) Microarray expression folds of DUSP6 (left). The expression of DUSP6 was cofirmed by real-time PCR ( $n=3$, middle) and Western blotting (right). Both Western blotting and real-time PCR experiment was well correlated with microarray analysis. (B) Western blot analyses demonstrating DUSP6 overexpressed in ECs transfected with full length DUSP6 gene plasmid pDUSP6. pCMV was the vector control. (C) Survival ratio of pDUSP6 transfection. The pCMV group was arbitrarily set at 1. (D) Apoptosis analysis of pDUSP6 transfection. The index of pCMV group was set at 1 . (E) Representative soft agar colony images of pDUSP6 transfection. (F) Soft agar colony efficiency of DUSP6 overexpression. The efficiency of pCMV transfection control was set at 1. Dash border line, clone D09; compound border line, clone D10 $(n=3)$. Student's t-test was used for significance statistics $(p<0.05$ or $\mathrm{p}<0.01$ ) between DUSP6 overexpression group (gray) and the vector control group (black).

\section{Discussion}

Our in vitro and in vivo experiments showed that tumorigenesis declined significantly after ECs-hMSCs fusion. The stem cell culture condition has no effect on the suppression, implying the suppression was from hMSCs not the treatment process. The tetraploidy model of carcinogenesis argued that fused cells can become cancer by tetraploid and chromosomal instability caused aneuploidy (25). For this reason, the self-fusion of hMSCs was inspected simultaneously and no tumor developed in near 2-month observation. A probable explanation is that the tetraploid hybrids were prone to undergo spontaneous apoptosis to limit tetraploidization in normal cells (26). This might explain the increased spontaneous apoptosis in our hybrids as well.
However, spontaneous apoptosis could not elucidate the phenomena that tumorigenesis ability was preserved by selffusion of EC cells. There must be some genetic or other molecular changes that contribute to fusion-induced suppression. Pathway enrichment suggested that cell cycle arrest was coupled with apoptosis and increased expression of DUSP6 in MAPK pathway might negatively regulate proliferation after fusion. We focused on DUSP6, which had previously been shown down-regulated in EC and lung cancer. Additionally it has a potential role in tumor growth and progression $(27,28)$. Our primary tissue investigation confirmed the level of DUSP6 was negatively related to the differentiation state of EC (unpublished data). Exogenous expression of DUSP6 induced significant apoptosis and growth suppression in ECs, indicating 
Table III. Tumor formation of exogenous DUSP6 overexpression.

\begin{tabular}{|c|c|c|c|c|c|c|c|c|c|c|}
\hline Transfection & Cell line & Plasmid & $\begin{array}{c}\text { DUSP6 } \\
\text { level }\end{array}$ & $\begin{array}{c}\text { Inoculate } \\
\text { cell no. }\end{array}$ & $\begin{array}{c}\text { Tumors/ } \\
\text { mice }\end{array}$ & Weight (mg) & Volume $\left(\mathrm{mm}^{3}\right)$ & $\begin{array}{l}\text { Tumor } \\
\text { latency } \\
\text { (days) }\end{array}$ & $\begin{array}{c}\text { Sacrifice } \\
\text { time } \\
\text { (days) }\end{array}$ & \\
\hline \multirow[t]{4}{*}{ Transient } & EC9706 & pCMV & - & $5 \times 10^{5}$ & $5 / 5$ & $566.9 \pm 298.3$ & $1025.5 \pm 482.2$ & $8.4 \pm 1.3$ & 26 & \\
\hline & & pDUSP6 & +++ & $5 \times 10^{5}$ & $2 / 5$ & $35.4 \pm 12.2$ & $31.8 \pm 8.4$ & $22.3 \pm 1.2$ & & a \\
\hline & KYSE150 & pCMV & - & $5 \times 10^{5}$ & $5 / 5$ & $429.1 \pm 260.9$ & $947.6 \pm 615.4$ & $17.2 \pm 1.1$ & 32 & \\
\hline & & pDUSP6 & +++ & $5 \times 10^{5}$ & $0 / 5$ & ND & ND & ND & & $\mathrm{b}$ \\
\hline \multirow[t]{5}{*}{ Stable clones } & EC9706C & pCMV & - & $5 \times 10^{5}$ & $6 / 6$ & $4501.9 \pm 751.5$ & $7777.8 \pm 2233.3$ & $8.5 \pm 1.6$ & & \\
\hline & CloneD09 & pDUSP6 & +++ & $5 \times 10^{5}$ & $5 / 5$ & $1216.1 \pm 436.6$ & $2092.1 \pm 1282.0$ & $11.2 \pm 1.1$ & 72 & $\mathrm{c}$ \\
\hline & Clone D10 & pDUSP6 & +++ & $5 \times 10^{5}$ & $6 / 6$ & $1678.8 \pm 555.9$ & $2322.3 \pm 1129.5$ & $11.3 \pm 1.0$ & & $\mathrm{c}$ \\
\hline & Clone D08 & pDUSP6 & + & $5 \times 10^{5}$ & $5 / 5$ & $1274.6 \pm 643.4$ & $1707.7 \pm 857.0$ & $9.4 \pm 6.4$ & 61 & \\
\hline & Clone D16 & pDUSP6 & +++ & $5 \times 10^{5}$ & $5 / 5$ & $1832.6 \pm 295.9$ & $1744.7 \pm 545.1$ & $9.2 \pm 4.0$ & & d \\
\hline
\end{tabular}

EC9706 C was the pCMV stable transfection. -, no expression; +, low expression; +++, high expression. ND, not detected. Wilcoxon signed ranks test, significant difference of tumor weights, volumes and the latency times between groups. ${ }^{\mathrm{a}, \mathrm{b}, \mathrm{p}} \mathrm{p}<0.05$ (pDUSP6 vs. pCMV group respectively); ${ }^{\mathrm{d}} \mathrm{p}<0.001$ (D16 vs. D08, whole group comparison with two-way ANOVA).

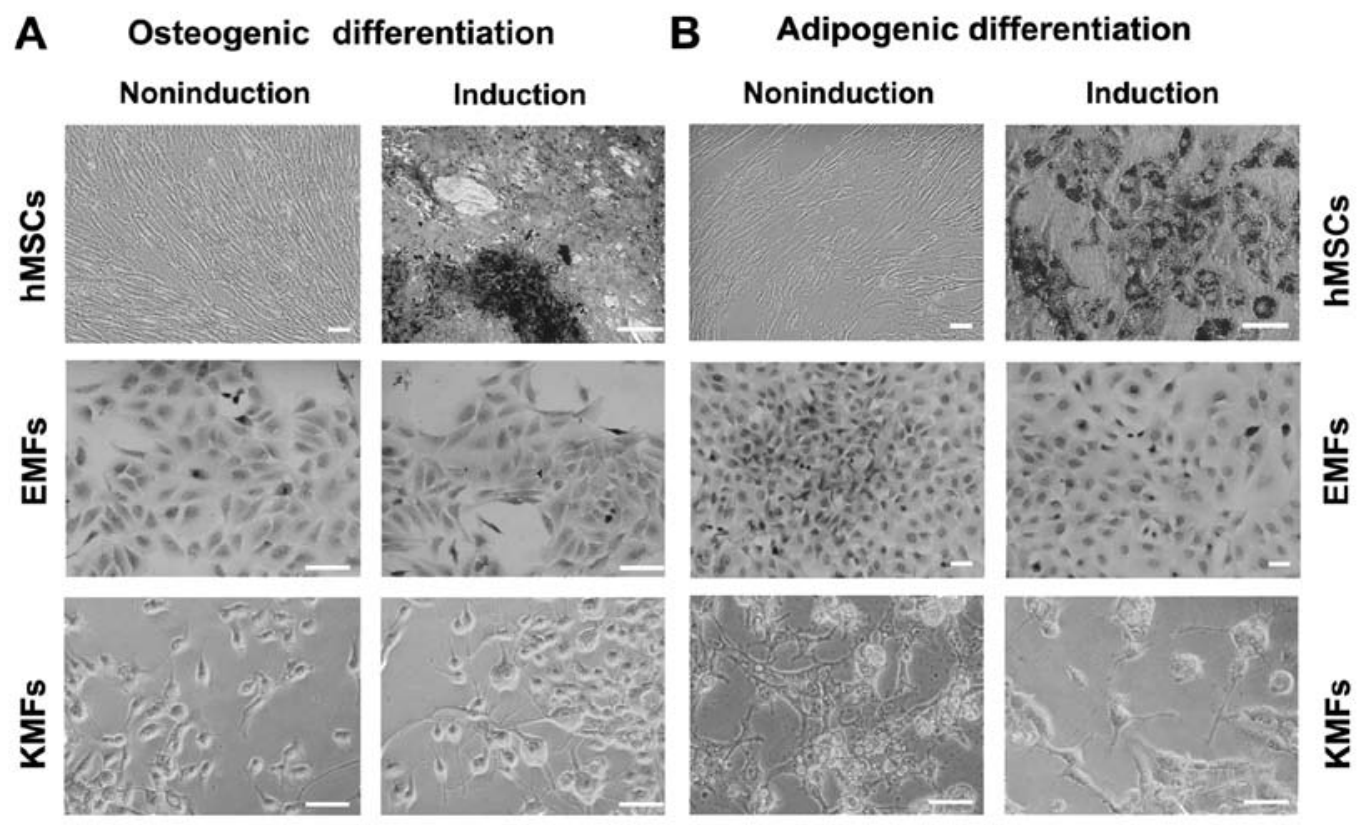

Figure 5. Osteogenic and adipogenic differentiation. (A) Osteogenic differentiation was evidenced by the formation of mineralized matrix in positive control hMSCs after osteogenic induction. No mineralized matrix formation was found in hybrids. Bars, $25 \mu \mathrm{m}$. Magnification, hMSCs non-induction x 200 , others x400. (B) Adipogenic differentiation was evidenced by the formation of lipid vacuoles with oil-red O staining in hMSCs after adipogenic induction. No lipid vacuoles were found in hybrids. Non-induction of hMSCs was the negative control. Bars: $25 \mu \mathrm{m}$, Magnification, hMSC non-induction, and all EMFs x200, others x400.

the involvement of DUSP6 in the pro-apoptotic pathway. Direct evidence has demonstrated exogenous expression of DUSP6 could induce pancreatic cancer cell apoptosis by ERK suppression and mediate AMPA receptor-induced oligodendrocyte death $(29,30)$, while down-regulation of DUSP6 mediates up-regulation of ERK and anti-apoptosis effect (31). All these implied that suppression of ERK by DUSP6 may induce apoptosis, which could also explain our results.
Cell fusion is one mechanism through which cells could acquire new properties and might contribute to cancer progression $(32,33)$. However, all the neutral characteristics discussed above indicated the hybrids only exhibit an intermediary phenotype transition. To our great surprise, there was a striking decline of tumorigenesis in all hybrids and the hybrids had no osteogenic or adipogenic potential, implying that hMSCs were not good candidates for pluripotency reprogramming. One main charac- 
teristic of cancer stem cells is strong tumorigenicity. However, our results showed the hybrids had declining tumorigenicity, suggesting cell fusion of hMSCs with ECs did not generate cancer stem cells.

In conclusion, our results demonstrate ECs-hMSCs fusion induced apoptosis and benign transdifferentiation rather than reprogramming to cancer stem cells. Our data also suggest DUSP6 has a tumor suppressor potential especially in transient overexpression circumstances. Our study provides new evidence for cell fusion of MSCs with tumor cells in tumorigenesis.

\section{Acknowledgements}

This work was supported by: National Basic Research Program of China (973 Program, no. 2009CB521803), National Natural Science Foundation of China (no. 30971448). We thank Dr Xiaohong Han for flow cytometry analysis, Dr Xun Zhang for histological evaluation of tumors, Professor Zhongchao Han (Institute of Hematology) for providing first batch of hMSCs and Dr Hong Lian (ILAS) for providing the human ES cell line. All authors declare that there is no conflict of interest in connection with this study.

\section{References}

1. Archuleta TD, Devetten MP and Armitage JO: Hematopoietic stem cell transplantation in hematologic malignancy. Panminerva Med 46: 61-74, 2004.

2. Smalley M and Ashworth A: Stem cells and breast cancer: a field in transit. Nat Rev Cancer 3: 832-844, 2003.

3. Kidd S, Spaeth E, Dembinski JL, et al: Direct evidence of mesenchymal stem cell tropism for tumor and wounding microenvironments using in vivo bioluminescent imaging. Stem Cell 27: 2614-2623, 2009.

4. Khakoo AY, Pati S, Anderson SA, et al: Human mesenchymal stem cells exert potent antitumorigenic effects in a model of Kaposi's sarcoma. J Exp Med 203: 1235-1247, 2007

5. Sasportas LS, Kasmieh R, Wakimoto H, Hingtgen S, van de Water JA and Mohapatra G: Assessment of therapeutic efficacy and fate of engineered human mesenchymal stem cells for cancer therapy. Proc Natl Acad Sci USA 106: 4822-4827, 2009.

6. Matsuzuka T, Rachakatla RS, Doi C, et al: Human umbilical cord matrix-derived stem cells expressing interferon-beta gene significantly attenuate bronchioloalveolar carcinoma xenografts in SCID mice. Lung Cancer 70: 28-36, 2010.

7. Ganta C, Chiyo D, Ayuzawa R, et al: Rat umbilical cord stem cells completely abolish rat mammary carcinomas with no evidence of metastasis or recurrence 100 days post-tumor cell inoculation. Cancer Res 69: 1815-1820, 2009.

8. Orimo A, Gupta PB, Sgroi DC, et al: Stromal fibroblasts present in invasive human breast carcinomas promote tumor growth and angiogenesis through elevated SDF-1/CXCL12 secretion. Cell 121: 335-348, 2005.

9. Mani SA, Guo W, Liao MJ, et al: The epithelial-mesenchymal transition generates cells with properties of stem cells. Cell 133: 704-715, 2008.

10. Li HC, Stoicov Calin, Liu JH and Houghton JM: Fusion of MSC with gastric epithelial cells increases invasion and metastasis of gastric cancer. Gastroenterology 140: S-39, 2011.

11. Bianco P: Mesenchymal stem cells: revisiting history, concepts, and assays. Cell Stem Cell 2: 313-319, 2008.
12. Harris H, Miller OJ, Klein G, Worst P and Tachibana T: Suppression of malignancy by cell fusion. Nature 223: 363-368, 1969.

13. Stanbridge EJ: Suppression of malignancy in human cells. Nature 260: $17-20,1976$

14. Anderson MJ and Stanbridge EJ: Tumor suppressor genes studied by cell hybridization and chromosome transfer. FASEB J 7: 826-833, 1993.

15. Vassilopoulos G, Wang PR and Russell DW: Transplanted bone marrow regenerates liver by cell fusion. Nature 422: 901-904, 2003.

16. Ying QL, Nichols J, Evans EP and Smith AG: Changing potency by spontaneous fusion. Nature 416: 545-548, 2002.

17. Spees JL, Olson SD, Ylostalo J, et al: Differentiation, cell fusion, and nuclear fusion during ex vivo repair of epithelium by human adult stem cells from bone marrow stroma. Proc Natl Acad Sci USA 100: 2397-2402, 2003.

18. Zhao P, Dai M, Chen W and Li N: Cancer trends in China. Jpn J Clin Oncol 40: 281-285, 2010.

19. He Z, Zhao Y, Guo C, et al: Prevalence and risk factors for esophageal squamous cell cancer and precursor lesions in Anyang, China: a population-based endoscopic survey. Br J Cancer 103: 1085-1088, 2010.

20. Pereira $\mathrm{CF}$ and Fisher AG: Heterokaryon-based reprogramming for pluripotency. Curr Protoc Stem Cell Biol Chapter 4: Unit 4B.1, 2009.

21. Lu LL, Liu YJ, Yang SG, et al: Isolation and characterization of human umbilical cord mesenchymal stem cells with hematopoiesis-supportive function and other potentials. Haematologica 91: 1017-1026, 2006.

22. Zhang C, Zhu C, Chen H, et al: Kif18A is involved in human breast carcinogenesis. Carcinogenesis 31: 1676-1684, 2010.

23. Livak KJ and Schmittgen TD: Analysis of relative gene expression data using real-time quantitative PCR and the 2(-Delta Delta C(T)) method. Methods 25: 402-408, 2001

24. Terada N, Hamazaki T, Oka M, et al: Bone marrow cells adopt the phenotype of other cells by spontaneous cell fusion. Nature 416: 542-545, 2002.

25. Margolis RL: Tetraploidy and tumor development. Cancer Cell 8: 353-354, 2005.

26. Castedo M, Coquelle A, Vivet S, et al: Apoptosis regulation in tetraploid cancer cells. EMBO J 25: 2584-2595, 2006.

27. Leung AC, Wong VC, Yang LC Y, et al: Frequent decreased expression of candidate tumor suppressor gene, DEC1, and its anchorage-independent growth properties and impact on global gene expression in esophageal carcinoma. Int J Cancer 122: 587-594, 2008

28. Okudela K, Yazawa T, Woo T, et al: Down-regulation of DUSP6 expression in lung cancer: its mechanism and potential role in carcinogenesis. Am J Pathol 175: 867-881 2009.

29. Furukawa T, Sunamura M, Motoi F, Matsuno S and Horii A: Potential tumor suppressive pathway involving DUSP6/MKP-3 in pancreatic cancer. Am J Pathol 162: 1807-1815, 2003.

30. Domercq M, Alberdi E, Sánchez-Gómez MV, Ariz U, PérezSamartín A and Matute C: Dual-specific phosphatase-6 (Dusp6) and ERK mediate AMPA receptor-induced oligodendrocyte death. J Biol Chem 286: 11825-11836, 2011.

31. Rössig L, Haendeler J, Hermann C, et al: Nitric oxide downregulates MKP-3 mRNA levels: involvement in endothelial cell protection from apoptosis. J Biol Chem 275: 25502-25507, 2000.

32. Ferrand J, Noël D, Lehours P, et al: Human bone marrow-derived stem cells acquire epithelial characteristics through fusion with gastrointestinal epithelial cells. PLoS One 6: e19569, 2011.

33. Cowan CA, Atienza J, Melton DA and Eggan K: Nuclear reprogramming of somatic cells after fusion with human embryonic stem cells. Science 309: 1369-1373, 2005. 